\title{
Acculturation Strategies Among Ethnic Minority Workers and the Role of Intercultural Personality Traits
}

\author{
Kyra Luijters, Karen I. van der Zee and Sabine Otten \\ University of Groningen
}

\begin{abstract}
In an increasingly diverse work context minority employees strive to place and define themselves in terms of work and cultural identities. Based on Berry's acculturation model (1990), we defined and tested preferred acculturation strategies at work. It was predicted that the dual identity, reflecting strong cultural identity maintenance combined with strong team identity adoption, is the most preferred strategy at work. The present study among non-Dutch employees working in The Netherlands $(N=108)$ showed that the dual identity is indeed preferred over strong team identity adoption, but solely among minority members who are emotionally stable. It is argued that these people are competent in dealing with the extra conflict and diversity-related stress that this acculturation strategy produces.
\end{abstract}

KEYWORDS diversity, acculturation at work, dual identity, emotional stability

THE NUMBER of employees with non-native cultural backgrounds has increased rapidly over the past decades. According to Chun (1983) '[Ethnic minority] individuals continually strive to place and define themselves in a world of relationships and meanings. [...]. The sense of identity emerges as an individual clarifies these issues and $\mathrm{s} /$ he learns to place oneself within the total configuration'. Ethnic minority workers need to decide how much emphasis they place on norms, values and habits that define their cultural and organizational identity. Finding that out for themselves is important for their well-being and work outcomes. In the present study, we will examine how ethnic minority workers prefer to define their identities within the work context. Mainly based on Berry's acculturation model (Berry, 1990), the present study aims to predict the preferred acculturation strategy of ethnic minority workers in Dutch organizations. In

\section{Author's note}

Address correspondence to Kyra Luijters, Department of Social \& Organizational Psychology, University of Groningen, Grote Kruisstraat 2/1, 9712 TS Groningen, The Netherlands [email: K.Luijters@rug.nl] 
addition, we were interested in the role of personality traits as moderators of different acculturation strategies.

The present study is important in many ways. Earlier research studied acculturation into general society, whereas, as far as we know, little research has been done with respect to acculturation in specific settings (e.g. Arends-Tóth \& van de Vijver, 2004). Studying acculturation in the work context is important, because patterns of identification of ethnic minority workers seem to be an important cause of the communication problems and conflicts that have often been associated with negative outcomes of cultural diversity in the literature (e.g. O'Reilly, Caldwell \& Barnett, 1989; Tsui, Egan \& O’Reilly, 1992). Knowledge about acculturation preferences of ethnic minority workers might teach us how to clear the way for more constructive intergroup relations at work, which might eventually contribute to often mentioned values of diversity, such as innovation and better decision making (e.g. McLeod, Lobel, \& Cox, 1996; Mumford \& Gustafson, 1988). Therefore, knowledge about preferred acculturation strategies offers points of application for organizations dealing with these diversityrelated problems.

Acculturation is defined as the mutual adaptation of behaviour and habits among people with different ethnic backgrounds. According to the acculturation theory of Berry and colleagues (e.g. Berry, 1990, 1997; Segall, Dasen, Berry, \& Poortinga, 1999), individual members must deal with the issue of how to acculturate, hence they usually work out strategies in their daily encounters with each other with respect to two major issues. The first major issue that Berry and colleagues defined is cultural maintenance, referring to the extent to which cultural (ethnic) identity and characteristics are considered to be important, and to which their maintenance is strived for. The second major issue that they defined is contact and participation. This issue concerns the extent to which immigrants choose to be involved in other cultural groups, or remain primarily among themselves.

Berry's model refers to acculturation of immigrants to a new society. We expect both dimensions not only to be relevant in general society, but also in a work context. In a work context, minority workers become part of a team which can be considered as a small society. In addition, because of differences in backgrounds, cultures are likely to become salient at work, and workers determine the extent to which they want to maintain their own culture that differs from others within their team.

On the other hand, there are differences between acculturation to a society and acculturation at work. Berry $(1990,1997)$ distinguishes between the old and the new culture, thereby referring to a clear in-group (the old culture) versus an out-group (the new, host culture). Because in the work context individuals are members of an entity, i.e. the team, in which they have to collaborate in order to reach common goals, this group cannot be regarded as an outgroup, but rather as a second in-group encompassing both minority and majority members. Second, in his original model, Berry (1990, 1997) contrasts identification with the original culture with contact with the new culture. In an organization, however, contact and participation with team members is an automatic result of the structural context. In that context, the amount of identification with the team seems to be a more important indicator of acculturation than the amount of contact with fellow team members. Contact and participation with the team are only relevant to the extent that they are freely chosen.

As a result, in our model we differentiate acculturation at work according to two dimensions (see Figure 1). The first dimension is cultural maintenance; the extent to which ethnic minority workers want to maintain their cultural identity and to which characteristic features of their culture are considered to be important. The second dimension is team identity adoption; the extent to which the work team and its characteristics are considered to be important, and their adoption strived for. Consequently, these two dimensions yield four acculturation strategies. First, the marginal identity is referred to as comprising of a weak need to maintain key features of one's own 


\section{$\underline{\text { Team Identity Adoption }}$}

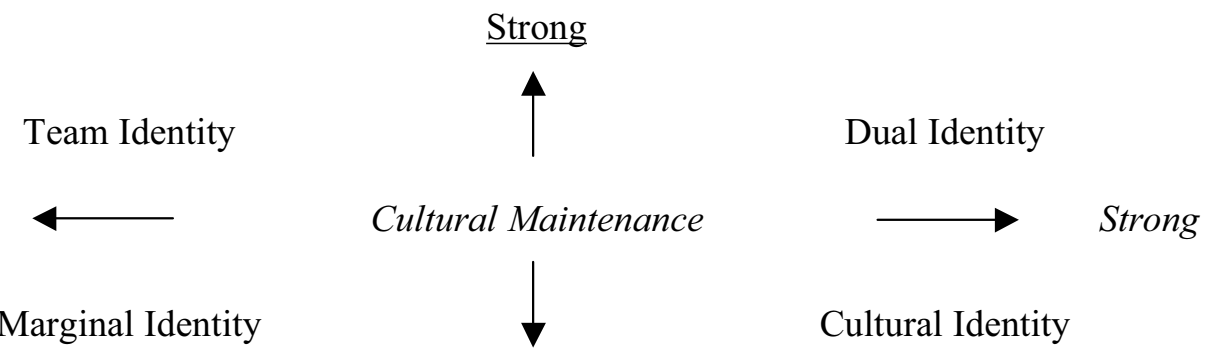

Weak

Figure 1. Acculturation strategies of employees with non-Dutch backgrounds at work.

culture, combined with a weak need to adopt key features of one's work team. Second, the cultural identity refers to the dominant need to maintain the key characteristics of one's own culture, while distancing from the key features of one's work team. Third, the team identity implies a need to distance oneself from key characteristics of one's cultural background, combined with a strong need to adopt key features of one's work team. Lastly, we will use the term dual identity to refer to the strategy in which strong maintenance of one's cultural background is combined with a strong identification with one's work team. The idea of dual identification is based on the recognition that different social identities at different levels of inclusiveness can be combined. In addition, dual identification has been promoted as an appropriate model for multiethnic societies (see also Berry, 1984; Brewer, 2001; Huo, Smith, Tyler, \& Lind, 1994).

Based on Berry's adapted framework, in the present study we examined which one of these acculturation strategies is preferred among ethnic minority employees working in Dutch organizations. Arends-Tóth and van de Vijver (2004) examined patterns of acculturation across different life domains. Their research suggests that Turkish-Dutch people find it most important to adopt the new culture in public domains, implying that they would adopt the team identity in the work domain. In addition, research shows that in general, most employees highly value strong team identity adoption (e.g. Hogg \& Terry, 2001). In the present study, we therefore expect that minority workers prefer strong team identity adoption to weak team identity adoption (Hypothesis 1a).

While minority employees highly value strong team identity adoption, they are raised with their cultural identity, therefore, they can hardly deny it. The values associated with the cultural identity are likely to be deeply engrained into the self. Moreover, the rules and norms shared within a culture provide a sense of belongingness and safety. Like many group memberships, affiliation with cultural backgrounds is often powerful with strong emotional meaning (Brown, Vivian, \& Hewstone, 1999). In addition, the cultural identity might be a source of self esteem (Phinney, 1990). Thus, there is evidence suggesting that cultural maintenance should also be preferred.

Although cultural identification is expected to affect evaluations of acculturation in the work context positively, we argue that this will only be the case when cultural maintenance is combined with strong team identity adoption. A sole emphasis of cultural identities at work might yield tensions and negative work outcomes, resulting for example from value conflicts and communication problems (e.g. Brickson, 2000; Van der Zee, Atsma, \& Brodbeck, 2004). On the other hand, people 
who adopt the dual identity become full members of the new group, without having to abandon their original cultural group. In line with this reasoning, research on acculturation has shown that ethnic minorities respond most positively to the integration strategy, and this strategy is associated with high well-being (e.g. Bakker, Van der Zee, \& Van Oudenhoven, 2003, in press; Berry, 1990; Berry, Kim, Power, \& Bujaki, 1989; Van Oudenhoven, Prins, \& Buunk, 1998). Similar conclusions can be drawn from literature on the benefits of a dual identity (Brewer, 2001; Gaertner, Rust, Dovidio, Bachman, \& Anastasio, 1994; Hornsey \& Hogg, $2000)$. Therefore, in the present study we predicted that strong team identity adoption, combined with strong cultural maintenance is the most preferred strategy among minority employees (Hypothesis 1b).

In conclusion, we expect a main effect of team identity adoption, and an interaction between team identity adoption with cultural maintenance. We do not postulate a main effect of cultural maintenance, as we expect the effect of cultural maintenance in a work context to be dependent on team identity adoption. As argued, we expect that cultural maintenance adds value under conditions of strong team identity adoption, but not under conditions of weak team identity adoption.

\section{Acculturation and intercultural personality traits}

We herein argue that the dual identity is the strategy that is evaluated most positively among ethnic minority workers. According to Berry (1991), however, although integration generally is the most preferred strategy, it is also a strategy that can yield stress when the majority group is not open and inclusive. Other studies also argued that immigrants who both try to maintain their cultural identity within the new environment and try to engage in contact with this new environment are subject to this diversity-related stress (Van Oudenhoven \& Eisses, 1998). When individuals try to combine both identities, tension between norms that are linked to each identity may give rise to role conflict (Gaertner et al., 1994). Moreover, in order to fit in the new group, the cultural identity needs to be negotiated with the new group members.

Consequently, we argue that although a dual identity might be the most preferred strategy at work, it requires that minority members are able to deal with the stress. Therefore, in the present study we examined to what extent traits that are linked to the ability to deal with acculturative stress determine the preference for acculturation strategies at work.

In the literature, personality traits have been related to intercultural success and to people's competence in coping with intercultural situations (Van der Zee \& Van Oudenhoven, 2000, 2001). Cultural Empathy refers to the ability to empathize with the feelings, thoughts and behaviors of members from different cultural groups. Open-mindedness refers to an open and unprejudiced attitude towards outgroup members and towards different cultural norms and values. Emotional Stability refers to a tendency to remain calm in stressful situations. Finally, Flexibility reflects the ability to switch easily from one strategy to another. Moreover, people scoring high on this dimension are not afraid of new and unknown situations but rather feel attracted to them, seeing them as a challenge rather than as a threat. ${ }^{1}$

We expect that these intercultural traits moderate the hypothesized interaction effect of team adoption and cultural maintenance on preferences for acculturation strategies. More precisely, we expect that the preference for the dual identity is especially pronounced among high scorers on these intercultural traits (Hypothesis 2).

\section{Method}

\section{Summary of the experimental design and procedure}

In the present study we were interested in how participants evaluated different acculturation strategies. Moreover, we investigated personality as a post hoc factor that could possibly influence participants' evaluation. To test our assumptions, we used the scenario technique, 
in which the participants evaluated a realistic example of a person and situation (e.g. Bakker et al., 2003; Van Oudenhoven et al., 1998). The fact that participants do not report their own behavior and respond to only one acculturation strategy, instead of comparing the four strategies, discourages social desirability. In the present study, participants read a scenario describing an interview with a male, Turkish employee, working for a Dutch organization. The scenario was presented as a newspaper article, and contained information corresponding to one of the four acculturation strategies (see appendix for the scenarios). Thus, a 2 (weak versus strong maintenance of cultural identity) $\times 2$ (weak versus strong adoption of team identity) between-subjects design was used. All questionnaires were in Dutch.

\section{Pilot study}

In order to check whether the manipulations were successful, we performed a pilot study among people with non-Dutch backgrounds, mostly from Iran and Turkey. In total, 12 cultural backgrounds were represented. Twenty participants (five per scenario) read a newspaper article about a Turkish employee named Ömer who works for a Dutch organization, and answered two questions related to the adoption of team identity ('Does Ömer feel part of his organization?' and 'Does Ömer's team take up an important place in his life?'), and two questions related to cultural maintenance ('Does Ömer stay close to his Turkish background?' and 'Does Ömer maintain his Turkish background?'). Participants answered on a 5-point scale ranging from 1 (not at all) to 5 (completely). A mean score of two questions per factor was computed (for the adoption of team identity $r=.94, p<.001$; for the maintenance of cultural identity $r=.91, p<.001)$. A multivariate analysis of variance (MANOVA) confirmed that the article successfully affected the perceived strength of both factors. Participants estimated Ömer's strength of team identity to be stronger in the strong team adoption condition $(M=4.50)$ than in the weak team adoption condition $(M=1.30 ; F(1,16)=210.1, p<.001)$. Moreover, participants estimated Ömer's strength of cultural maintenance to be stronger in the strong cultural maintenance condition $(M=3.85)$ than in the weak cultural maintenance condition $(M=1.50 ; F(1,16)=105.2, p<$ $.001)$. Moreover, no significant interaction emerged with respect to the estimated strength of cultural maintenance $(F(1,16)=3.86, p=$ $.067)^{2}$ nor for the estimated strength of team adoption $(F<1)$.

\section{Main study}

Participants Participants were employees with non-Dutch backgrounds, who were employed in organizations in The Netherlands. The questionnaires were distributed via both profit and non-profit agencies that helped people with non-Dutch backgrounds to enter and remain in the job market. In total, 400 questionnaires were distributed; 108 questionnaires were returned, resulting in a response rate of $27 \%$. The possible explanations for this low response rate are presented in the Discussion section. In total, we received 27 questionnaires with the dual identity scenario, 25 with the team identity scenario, 31 with the cultural identity scenario and 25 with the marginal identity scenario. Participation was voluntary for all employees and confidentiality of responses was assured.

Participants had different cultural backgrounds. Most participants were from Surinam (25 participants) and Turkey (30 participants). Sixteen other participants were born in The Netherlands but had parents who were born in another country. In total, 23 countries were represented in our sample, including Indonesia, Morocco, Netherlands Antilles and Iran. Slightly more women than men participated $(54 \%)$. On average, participants were 40 years old $(S D=10.21)$, and had lived in The Netherlands for 24 years $(S D=10.47)$. Most participants had received higher level $(88 \%)$ or middle level $(10 \%)$ education. On average, they had been employed in The Netherlands for 13.77 years $(S D=10.56)$, and they had worked in their present job for 6.19 years $(S D=$ 7.32 years). 
Dependent measures Right after the participants read the scenario, they were asked questions to check the credibility of the scenario. Next, we measured respondents' evaluation of the scenario target, followed by the Multicultural Personality Questionnaire and a demographic questionnaire.

Credibility of the scenario To check whether participants believed that the fictitious newspaper article was real, we asked the participants in which newspaper they thought the article had appeared. In response to this question, most participants referred to an existing newspaper (90 participants), 13 participants didn't answer, four participants said they didn't know, and only one respondent claimed that it was a fictitious article. This respondent was removed from the analyses.

Evaluation of the scenario target Three questions indicated participants' affective reactions to the target, namely: 'Does this article about Ömer bring up negative feelings in you?' (-); 'Does this article about Ömer bring up positive feelings in you?'; and 'How sympathetic do you think Ömer is?' Participants answered on a 5-point scale, ranging from 1 (not at all) to 5 (very much). One question indicated participants' normative reaction to the target, namely: 'Should nonnatives behave like Ömer?' Finally, two items indicated identification with the target, namely: 'Would you like to be like Ömer?' and 'Do you recognize yourself in Ömer?' Participants answered these three questions on a 5-point scale, ranging from 1 (not at all) to 5 (completely). Factor analysis with varimax rotation showed that these six items loaded on one factor (eigenvalue 3.98 , explaining $66 \%$ of the variance), indicating that the participants did not make the distinction between the different kinds of reactions. Therefore, we chose to make one scale that reflected their target evaluation ( $M$ $=2.86, S D=.85, \alpha=.89$ ).

Four scales from the Multicultural Personality Questionnaire (Van der Zee \& Van Oudenhoven, 2000, 2001) were used to measure the personality traits that indicate competence in coping with intercultural situations. Cultural
Empathy $(M=3.95, S D=.49, \alpha=.90)$ was measured with 18 items (e.g. 'Is interested in other cultures' and 'Feels uncomfortable in another culture (-)'). Open-mindedness $(M=$ $3.72, S D=.49, \alpha=.88)$ was measured with 18 items (e.g. 'Tries to understand other people's behavior' and 'sympathizes with others'). Emotional Stability $(M=3.66, S D=.43, \alpha=.83)$ was measured with 20 items (e.g. 'Keeps calm at ill-luck' and 'Is nervous (-)'). Finally, Flexibility $(M=.18, S D=.46, \alpha=.78)$ was measured with 18 items (e.g. 'Changes easily from one activity to another' and 'Works according to a plan (-)'). ${ }^{3}$ Participants were asked to indicate to what extent the items were applicable to themselves on a 5-point scale, ranging from 1 (not at all) to 5 (completely).

\section{Results}

The article always described a male, Turkish employee. As a result, Turkish and/or male participants might have evaluated the target differently than non-Turkish and/or female participants. Therefore, we checked for the main effects of these background variables and interactions with the factors within an ANOVA. However, main effects and interactions were nonsignificant, revealing no demographic influences. $^{4}$

\section{Evaluations of the target}

We expected that target evaluations would be more positive under conditions of strong team identity adoption as opposed to weak team identity adoption (Hypothesis 1a). Moreover, we expected an interaction between team identity adoption and cultural maintenance. That is, cultural maintenance is expected to contribute positively to the evaluation of the target under conditions of strong team identity adoption (Hypothesis 1b). These hypotheses were tested with an ANOVA with cultural maintenance and team identity adoption as independent dummy-coded variables. The target evaluation was entered in the analyses as the dependent variable. A significant main effect was found for team identity adoption $(F(1,103)$ $\left.=38.80, p<.001, \eta^{2}=.27\right)$. As predicted in 
Hypothesis 1a, the participants evaluated the target more positively under conditions of strong team identity adoption than weak team identity adoption $(M=3.33$ vs. $M=2.46)$. Unexpectedly, a significant main effect was also found for cultural maintenance $(F(1,103)=$ $\left.4.61, p<.05, \eta^{2}=.04\right)$. Participants evaluated the target more positively in the strong cultural maintenance condition than in the weak cultural maintenance condition $(M=3.00$ vs. $M=2.72$ ).

A marginally significant interaction effect between team identity adoption and cultural maintenance confirmed Hypothesis $1 \mathrm{~b}$ $\left(F(1,107)=3.55, p=.06, \eta^{2}=.03\right)$ (see Figure 2). Post hoc tests showed that in the strong team identity adoption condition, the target evaluations were more positive when the target strongly, as opposed to weakly, maintained his cultural identity $(M=3.59$ versus $M=3.03$; $F(1,103)=7.77, p<.01)$. However, in the weak team identity adoption condition, participants' evaluation of the target did not differ as a function of cultural maintenance $(M=2.47$ versus $M=2.44, F<1)$. We conclude that, as expected, strong cultural maintenance contributes positively to the evaluation of the target under conditions of strong team identity adoption.

\section{The role of intercultural traits on target evaluations}

We hypothesized that the two-way interaction effect as described earlier would be stronger for

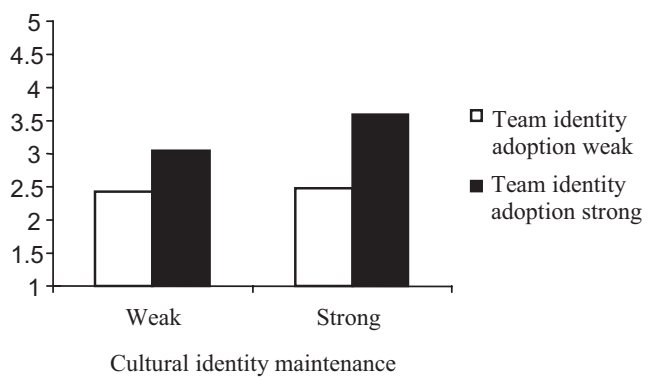

Figure 2. Evaluation of the target, ranging from 1 (negative) to 5 (positive) as a function of team adoption and cultural maintenance. individuals with high scores on intercultural traits (Hypothesis 2). This hypothesis was tested with an ANOVA with cultural maintenance and team identity adoption as independent dummycoded variables and the intercultural traits as covariates. The intercultural traits were continuous variables, which we standardized to prevent multicollinearity (Aiken \& West, 1991). Target evaluation was the dependent variable. Because of the complexity of the model, we analyzed the effects of the intercultural traits separately, starting with Cultural Empathy.

No main effect of Cultural Empathy on target evaluation appeared $(F(1,99)=2.91$, $p=.091$ ), but the interaction between Cultural Empathy and cultural maintenance was significant $\left(F(1,99)=5.59, p=.020, \eta^{2}=.05\right)$. To test the effect of this two-way interaction, contrasts were computed by filling in -1 and 1 for low and high levels of Cultural Empathy. This corresponds to one standard deviation below (low) and above (high) the mean for Cultural Empathy. Results show that for high levels of Cultural Empathy, target evaluation was more positive under conditions of strong cultural maintenance $(B=.63 ; F(1,99)=9.81, p=.002$, $\left.\eta^{2}=.09\right)$. However, for low levels of Cultural Empathy, target evaluation did not differ as a function of cultural maintenance $(B=-.06 ; F<$ $1)$.

With respect to Open-mindedness, neither significant main effects, nor interaction effects emerged $(p>.05)$. With respect to Flexibility, a main effect of Flexibility on target evaluation was found $\left(F(1,99)=4.37, p=.039, \eta^{2}=.04\right)$. However, it was only a weak effect as shown by a nonsignificant weight $(B=-.17 ; t<1)$. No significant interaction effects emerged $\left(F_{\mathrm{s}} \leq\right.$ $1.20, p \geq .28$ ).

In addition, a main effect of Emotional Stability was found $(F(1,99)=9.14, p=.003$, $\left.\eta^{2}=.08\right)$. The two-way interaction between cultural maintenance and team identity adoption was also significant $(F(1,99)=6.94$, $\left.p<.01, \eta^{2}=.07\right)$. More importantly, in line with our hypothesis, a significant three-way interaction emerged between cultural maintenance, team identity adoption and Emotional Stability $\left(F(1,99)=8.27, p=.005, \eta^{2}=.08\right)$. 
To illustrate the meaning of this three-way interaction effect, we tested the contrasts between the different conditions with post hoc analyses in ANOVA. In addition, we used regression analyses to compute the mean scores of target evaluation in every condition. By using the regression equation, mean scores can be computed for target evaluation in all eight conditions. Figure 3 shows that among participants high in Emotional Stability, in the strong team identity adoption condition, participants' target evaluation was more positive when the target strongly, as opposed to weakly, maintained his cultural identity $(M=3.65$ versus $M=2.57 ; F(1$, $\left.99)=16.18, p<.001, \eta^{2}=.14\right)$. In the weak team identity adoption condition, participants' target evaluation did not differ as a function of cultural maintenance ( $M=2.19$ versus $M=2.36$; $F(1,99)<1, p=.52)$. Among participants low on Emotional Stability, neither a main effect of cultural identity maintenance $(F(1,99)=2.41$, $p=.12)$, nor an interaction with team identity adoption $(F<1)$ was found. The main effect of team identity adoption was significant $(F(1,99)$ $\left.=15.91, p<.001, \eta^{2}=.14\right)$. In the strong team identity conditions, participants' target evaluation was positive, regardless of strong or weak cultural maintenance ( $M=3.54$ and 3.38 respectively) $(F<1)$. In the weak team identity adoption condition, participants' target evaluation was less positive, regardless of strong or weak cultural maintenance $(M=2.93$ and 2.51 respectively) $\left(F(1,99)=2.20, p=.14, \eta^{2}=.02\right)$.
We conclude that, among respondents low in Emotional Stability, the target evaluation depends on team identity adoption only.

\section{Discussion}

The present research aims to give more insight into preferred acculturation strategies of minority employees at work, and the role of intercultural personality traits as a determinant of this preference. As expected, the results showed that the minority employee who strongly identifies with his team is evaluated positively. This is in line with previous research that argued that both the organization and the work teams form important parts of employees' self definition (Hogg \& Terry, 2001), and found that Turkish-Dutch people value adaptation in public domains (Arends-Tóth \& van de Vijver, 2004).

The main prediction of this study was, however, that individuals particularly prefer a strong team identity if it comes together with a strong cultural identity. This prediction was also supported by our data, but it was found to hold solely for participants high on Emotional Stability. As we previously argued, combining identities can be stressful as the tension between norms linked to each identity may give rise to role conflict (Gaertner et al., 1994). Employees who clearly define and present themselves to colleagues as members of their cultural background probably encounter more

\section{Low Emotional Stability}

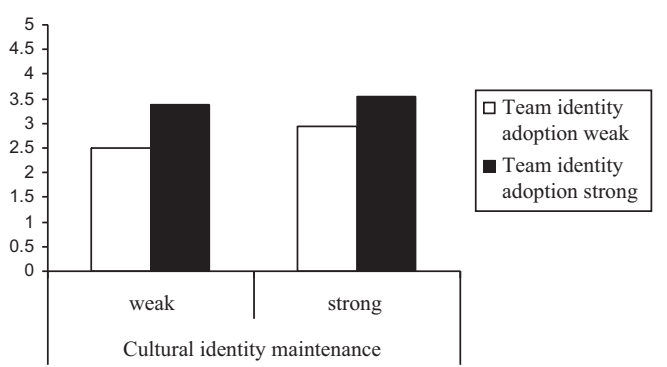

\section{High Emotional Stability}

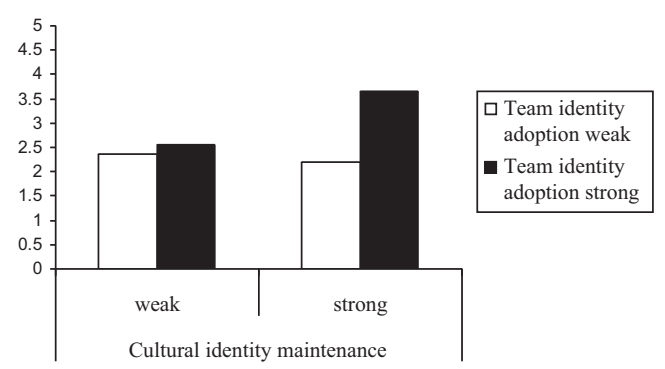

Figure 3. Target evaluation, ranging from 1 (negative) to 5 (positive) as a function team identity adoption and cultural identity maintenance for low and high emotional stability. 
diversity-related stress (Van Oudenhoven \& Eisses, 1998). Therefore, emotionally stable people adopt a strong team identity, retaining their cultural identity at the same time, as this identity and its values are likely to be deeply engrained into the self, as people are raised with them (Brown et al., 1999).

Unexpectedly, the present study also showed a main effect of cultural maintenance. Strong cultural maintenance was evaluated more positively than weak cultural maintenance. As cultural values are an important part of the self, it is not surprising that maintenance of these values is evaluated positively. In line with Bakker (2005) it can be argued that it seems generally desirable to maintain aspects of one's original culture. ${ }^{5}$ Yet, as expected, this main effect was qualified by an interaction with team identity, revealing that when combined with team identification, cultural maintenance is perceived most positively.

Noteworthy in this context is also the moderation effect of Cultural Empathy. We found that participants' scores on Cultural Empathy moderated the impact of cultural maintenance. For participants scoring high on Cultural Empathy, target evaluation was more positive when cultural maintenance was strong. For participants scoring low on Cultural Empathy, however, their target evaluation was independent of cultural maintenance. As Cultural Empathy refers to the capacity to sympathize with people with different beliefs, norms and values, it can well be that high scorers show more consideration with cultural maintenance, regardless of their own preference.

The expectation that high scorers of Cultural Empathy and Open-mindedness would show a preference for the dual identity, was not supported. The assumption of our study was that the preference for a dual identity among individuals high on the intercultural traits stems from their ability to deal with intercultural situations. Obviously, the issue of cultural maintenance is not specifically dealing with an intercultural situation, but has more to do with dealing with stress. Other research does indeed suggest that the ability to deal with stress and uncertainty are more closely linked to traits indicating low anxiety, such as Emotional Stability and Flexibility and not to traits indicating social curiosity, like Open-mindedness and Cultural Empathy (Van der Zee \& Van der Gang, 2006).

However, we must note that our results were not confirmed for Flexibility. We expected that Flexibility would be related to the competence to deal with diversity-related stress, because it reflects competence to change easily from one strategy to another. A recent study among Dutch immigrants on acculturation in general society showed that Flexibility is positively related to strategies indicating high contact, but negatively related to strategies indicating strong maintenance (Bakker, 2005). This suggests that Flexibility is related to a preference for the assimilation strategy. For future studies it would be interesting to find out whether the results of Bakker (2005) can be adapted to and replicated in the work context. Possibly, Flexibility is not related to competence in dealing with stress accompanied with combining two identities at work, because flexible people let go of their cultural identity easily.

Although the dual identity might be preferred as an ideal in a scenario, it may not always be feasible in real organizations. A dual identity implies that the majority accepts the minorities' quest to maintain their cultural identity (Segall et al., 1999). Therefore, the dual identity might be more feasible when the majority facilitates the formation of a dual identity among minority members. Future studies may focus on this issue. After all, cultural maintenance among minority members might be useful for organizations. Research suggests that embracing cultural identities helps to enhance creativity, and innovation in organizations (e.g. McLeod et al., 1996; Mumford \& Gustafson, 1988). Interestingly, some research has already been done focusing on context factors within organizations that support expression of different cultural identities within organizations. Recently, Van Knippenberg and Haslam (2003) suggested that within cultural diverse teams, a norm supporting diversity enhances identification. Furthermore, there is initial evidence showing that 
embracing different cultural identities is facilitated by means of an intercultural group climate (Harquail \& Cox, 1993), in which differences are valued, few prescriptions for behavour are defined and ambiguities are tolerated. This intercultural group climate is positively related to identification with the organization (Luijters, Van der Zee, \& Otten, 2006).

\section{Strengths, possible limitations and further implications}

Even though we received support for our main hypotheses, we need to acknowledge that our study has a number of possible limitations. Participants already worked in The Netherlands for a long time and had high levels of education. They can therefore be regarded as successful immigrants, having a job and good relations with team members in a country where unemployment among immigrants is high. Therefore, we think it is important to extend the study to more recent immigrants and newcomers in organizations. Despite our limited sample, however, we would argue that our findings yield important insights for organizations. Although the evaluation of strong team identity adoption may be biased in a positive direction among the present sample, there is no need to expect the influence of Emotional Stability on a preference for a dual identity to decrease among samples consisting of less successful emigrants. Hence, considering the higher level of stress that these emigrants may experience in trying to combine both entities makes the importance of this trait likely to be stronger rather than weaker than was found in the present study.

The low response rate can be regarded as another weakness of this study. Only $27 \%$ of the respondents that were approached participated. This is a common problem in research among ethnic minorities (Dinsbach, 2005). Ethnic minorities seem less familiar with the kind of survey research that is common in the Western part of the world (van 't Land, 2000). One explanation is that people want concrete results, but this kind of research does not have a direct impact on the situation of the participants them- selves (Meloen \& Veenman, 1990). Another explanation for the low response rate might be that language proficiency was too low to enable participation (Dinsbach, 2005; Van de Vijver, 2001). For future studies, it might be necessary to translate questionnaires. Filling in a questionnaire in the native language probably has the advantage that people can better distinguish between items that are conceptually close (Shadid, 1979). In our study, participants did not distinguish between the scales of affective reactions, normative reactions and identification. For future studies, these scales might be translated, or otherwise improved, to enable the conceptual distinction between the different outcome measures.

One strength of the study is that differences cannot be attributed to sample characteristics due to random assignment. Yet, it could be an interesting question to find out whether different samples do lead to diffent acculturation strategies. Do preferences differ for people with different backgrounds? Some research already suggests that the effectiveness of the different acculturation strategies differs for different cultural groups (e.g. Van Oudenhoven, Willemsma, \& Prins, 1996).

Another advantage of our method is that, compared to self-descriptions, the scenario method may decrease the tendency to answer in a socially desirable way, because participants respond to a realistic example of a person, instead of a direct question concerning their attitudes toward their own situation. Using a between-subjects design in which each participant receives only one scenario version further discourages social desirability. In this way, participants were unable to compare items or scenarios and evaluate one item more positively than the other because it is believed to be socially more desireable (Bakker, 2005). Despite this advantage of our method, critical readers could argue that evaluations of a target might differ from participant's own preferences. As we included a number of questions in this study, referring to participants' own identification with the team and cultural background, we were able to check this. Additional analyses confirmed our implicit assumption 
that participants' target evaluation corresponded to the strength of their own identification with their culture and their team, respectively.

With respect to our cultural maintenance manipulation, we want to note that this dimension was manipulated in a general way, as was done in previous scenario studies on acculturation (Bakker, et al., 2003; Van Oudenhoven et al., 1998). It seems logical to infer that, as the focus was on the work context, participants link general cultural maintenance to the work context. However, it would be interesting to phrase cultural maintenance at work more specifically.

In conclusion, the present study showed that employees with a cultural minority background have a preference for strong team identity adoption over strong cultural maintenance. In addition, the present study showed that only employees with minority backgrounds that score high on Emotional Stability preferred strong team identity adoption in combination with strong cultural identity maintenance over the other acculturation strategies. This suggests that a preference for the dual identity is dependent on the competence to deal with diversityrelated stress. The results of this study imply that in order to profit from diversity and cope with its problematic sides, organizations may work on a shared team identity. Furthermore, attempts to additionally promote maintenance of cultural identities should be accompanied by interventions aimed at reducing uncertainty and diversity-related stress.

\section{Notes}

1. Social Initiative, the tendency to actively approach social situations and to take initiative, is left out in the present study, as it refers to behavioral intentions, and is therefore less applicable to the evaluative reactions that we investigated in the present study.

2. This interaction is marginally significant, therefore we checked whether the trend in this interaction would be problematic for our manipulation. Contrast analysis showed that the effect of cultural maintenance is somewhat smaller under conditions of strong team identity adoption, as opposed to weak team identity adoption $(M=3.5$ versus $4.2, p<.05)$. This means that strong cultural maintenance was perceived as more extreme in the cultural identity condition as opposed to the dual identity condition. As the main effect of cultural maintenance is still strong under conditions of strong team identity adoption, and we are mostly interested in the effect of cultural maintenance under conditions of strong team identity adoption, in our opinion this marginal interaction is not problematic.

3. As this questionnaire was validated before and our $N$ might be too small to conduct a factor analysis on 74 items, we used the original scales. Although the correlation between Cultural Empathy and Open-mindedness is high $(r=.75$, $p<.001)$ and other correlations are moderate $(.29 \leq r \leq .51)$, they are not exceptional and earlier research shows that these traits do have distinct effects (Van der Zee \& Van Oudenhoven, 2000, 2001).

4. In addition, main and interaction effects were tested for gender, age, years in The Netherlands, tenure in organization, tenure in current job, tenure in current occupation and level of education. Separate ANOVAs showed no significant main effects nor interactions $(p>.10)$, except for a main effect of years in The Netherlands $(F(1,99)=5.52, p<.05)$. In line with the modest negative correlation of $-.18, p=.06$, this result shows that non-native people who lived in The Netherlands for a longer period of time evaluated Ömer slightly more negatively.

5. In research, negative effects of cultural maintenance were also found (see for instance Van der Zee et al., 2004), however, these studies have different dependent variables, for instance, well-being and commitment. We find the ambiguity intriguing, but not that surprising as our finding is consistent with other studies using the same dependent measures: in terms of preferences cultural maintenance might be preferable, in terms of work outcomes, it might be problematic.

\section{Acknowledgments}

Research was supported by a grant from the Dutch Science Foundation (NWO). We thank Femke Bekkema, Niek Faber, Dirk-Jacob Lemstra and Heleen Paagman for collecting most of the data. We thank the various organizations, especially 'Stichting Diversiteit \& Arbeid' who contacted the participants. We thank Winny Bakker, Maria Peeters, Nurcan 
Ensari and four anonymous reviewers for their helpful comments on earlier versions of this paper.

\section{References}

Aiken, L. S., \& West, S. G. (1991). Multiple regression: Testing and interpreting relations. Thousand Oaks, CA: Sage.

Arends-Tóth, J., \& van de Vijver, F. (2004). Domains and dimensions in acculturation: Implicit theories of Turkish-Dutch. International Journal of Intercultural Relations, 28, 19-35.

Bakker, W. (2005). Emigration and well-being. The role of personality and cultural identity in acculturation. Dissertation, University of Groningen, The Netherlands.

Bakker, W., Van der Zee, K. I., \& Van Oudenhoven, J. P. (2003). Individuele verschillen in reacties van migranten ten opzichte van culturele adaptatie [Individual differences in migrants' attitudes toward cultural adaptation]. Nederlands Tijdschrift voor de Psychologie, 58, 81-94.

Bakker, W., Van der Zee, K. I., \& Van Oudenhoven, J. P. (in press). Personality and dutch emigrants' reactions to acculturation strategies. Journal of Applied Social Psychology.

Berry, J. W. (1984). Cultural relations in plural societies: alternatives to segregation and their sociopsychological implications. In N. Miller \& M. Brewer (Eds.), Groups in contact: The psychology of desegregation (pp. 11-27). New York: Academic Press.

Berry, J. W. (1990). Psychology of acculturation. In J. Berman (Ed.), Cross-cultural perspectives: Nebraska symposium on motivation (pp. 201-234). Lincoln, NE: University of Nebraska Press.

Berry, J. W. (1991). Understanding and managing multiculturalism. Psychology and Developing Societies, 3, 17-49.

Berry, J. W. (1997). Immigration, acculturation and adaptation. Applied Psychology: An International Review, 46, 5-68.

Berry, J. W., Kim, U., Power, M, \& Bujaki, M. (1989). Acculturation attitudes in plural societies. Applied Psychology: An International Review, 38, 185-206.

Brewer, M. (2001). Toward reduction of prejudice: Intergroup contact and social categorization. In R. Brown \& S. L. Gaertner (Eds.), Blackwell handbook of social psychology: Intergroup processes. Oxford, UK: Blackwell.

Brickson, S. (2000). The impact of identity orientation on individual and organizational outcomes in demographically diverse settings. Academy of Management Review, 25, 82-101.

Brown, R., Vivian, J., \& Hewstone, M. (1999). Changing attitudes through intergroup contact: The effects of group membership salience. European Journal of Social Psychology, 29, 741-764.

Chun, K.-T. (1983). Ethnicity and ethnic identity: Taming the untamed. Cited in N. Hutnik (1991). Ethnic minority identity. A social psychological perspective (p. 20). Oxford, UK: Clarendon Press.

Dinsbach, A. A. (2005). Socialization in the workplace: A focus on migrant groups. Dissertation, Vrije University Amsterdam, The Netherlands.

Gaertner, S. L., Rust, M. C., Dovidio, J. F., Bachman, B. A., \& Anastasio, P. A. (1994). The contact hypotheses: The role of a common ingroup identity on reducing intergroup bias. Small Group Research, 25, 224-249.

Harquail, C. V., \& Cox, T. C. (1993). Organizational culture and acculturation. In T. Cox, Jr. (1993). Cultural diversity in organizations. Theory, research $\mathcal{E}$ practice (pp. 161-176). San Francisco: Berrett-Koehler.

Hogg, M. A., \& Terry, D. J. (2001). Social identity processes in organizational contexts. Philadelphia: Psychology Press.

Hornsey, M. J., \& Hogg, M. A. (2000). Assimilation and diversity: An integrative model of subgroup relations. Personality and Social Psychology Review, 4, 143-156.

Huo, Y., Smith, H., Tyler, T., \& Lind, E. A. (1994). Superordinate identification, subgroup identification, and justice concerns: Is separatism the problem, is assimilation the answer? Psychological Science, 7, 40-45.

Luijters, K., Van der Zee, K. I., \& Otten, S. (2006). Cultural diversity in organizations: Enhancing identification by overcoming differences. Manuscript in preparation.

McLeod, P. L., Lobel, S. A., \& Cox, T. H. (1996). Ethnic diversity and creativity in small groups. Small Group Research, 27, 248-264.

Meloen, J. D., \& Veenman, J. (1990). Het is maar de Vraag. ..., Onderzoek naar Responseffecten bij Minderhedensurveys [That is the question..., research on response effects among minority surveys]. Lelystad: Koninklijke Vermande.

Mumford, M. D., \& Gustafson, S. B. (1988). Creativity syndrome: integration, application, and innovation. Psychological Bulletin, 103, 27-43.

O’Reilly, C. A., Caldwell, D. F., \& Barnett, W. P. (1989). Work group demography, social integration, and turnover. Administrative Science Quarterly, 34, 21-37. 
Phinney, J. (1990). Ethnic identity in adolescents and adults: Review of research. Psychological Bulletin, 108, 499-514.

Segall, M. H., Dasen, P. R., Berry, J. W., \& Poortinga, Y. H. (1999). Human behaviour in global perspective. An introduction to cross-cultural psychology (p. 305). Boston, MA: Allyn and Bacon.

Shadid, W. A. (1979). Moroccan workers in the Netherlands. Dissertation, University of Leiden, The Netherlands.

Tsui, A. S., Egan, T. D., \& O’Reilly, C. A. (1992). Being different: Relational demography and organizational attachment. Administrative Science Quarterly, 37, 549-579.

Van der Zee, K. I., Atsma, N., \& Brodbeck, F. (2004). The influence of social identity and personality on outcomes of cultural diversity in teams. Journal of Cross-Cultural Psychology, 35, 283-303.

Van der Zee, K. I., \& Van der Gang, I. (2006). Personality and threat as moderators of reactions to cultural diversity in teams. Manuscript under review.

Van der Zee, K. I., \& Van Oudenhoven, J. P. (2000). The Multicultural Personality Questionnaire: A multidimensional instrument of multicultural effectiveness. European Journal of Personality, 14, 291-309.

Van der Zee, K. I., \& Van Oudenhoven, J. P. (2001). The Multicultural Personality Questionnaire: Reliability and validity of self- and other ratings of multicultural effectiveness. Journal of Research in Personality, 35, 278-288.

Van de Vijver, F. (2001). Psychologie en de multiculturele samenleving [Psychology and the multicultural society]. Inaugural lecture, Katholieke Universiteit Brabant, Tilburg, The Netherlands.

Van Knippenberg, D., \& Haslam, S. A. (2003). Realizing the diversity dividend. Exploring the subtle interplay between identity, ideology, and reality. In S. A. Haslam, D. van Knippenberg, M. J. Platow, \& N. Ellemers (Eds.), Social identity at work. Developing theory for organizational practice. New York: Psychology Press.

Van Oudenhoven, J. P., \& Eisses, A. M. (1998). Integration and assimilation of Moroccan immigrants in Israel and The Netherlands. International Journal of Intercultural Relations, 22, 293-307.
Van Oudenhoven, J. P., Prins, K. S., \& Buunk, B. P. (1998). Attitudes of minority and majority members towards adaptation of immigrants. European Journal of Social Psychology, 28, 995-1013.

Van Oudenhoven, J. P., Willemsma, G., \& Prins, K. S. (1996). Integratie en assimilatie van Marokkanen, Surinamers en Turken in Nederland [Integration and Assimilation of Moroccan, Surinamese and Turkish people in The Netherlands]. De Psycholoog, 31, 468-471.

van 't Land, H. (2000). Similar questions, different meanings. Dissertation, Vrije University, Amsterdam, The Netherlands.

Paper received 9 February 2005; revised version accepted 27 January 2006.

\section{Biographical notes}

KYRA LUijTers is a PhD student in social and organizational psychology at the University of Groningen, The Netherlands. Her research interests are in acculturation at work and the effects of cultural diversity on team identity and work outcomes. In addition, she is interested in uncertainty, organizational climate factors and personality as moderators of diversity outcomes.

KAREN I. VAN DER ZEE is Professor of organizational psychology and Director of a research institute on Integration and Social Resilience at the University of Groningen, The Netherlands. Her current research interest is in the influence of personality, threat, and social identities on work outcomes in culturally diverse teams. More generally, she is interested in acculturation processes and integration of migrant groups into host societies.

SABine otten is Associate Professor at the University of Groningen, The Netherlands. Currently, her main research interests are the cognitive and emotional determinants of ingroup favoritism and outgroup derogation and the role of social identities in aggressive interactions. Moreover, she is investigating the link between the individual and collective self, and the interplay between inter- and intragroup processes. 


\section{Appendix}

Translated scenarios italics have been added to stress the sentences that vary over conditions

\section{The dual identity}

\section{The multicultural workforce}

Amsterdam - The Dutch labor market is becoming more and more diverse. Over past decades many foreigners have come to The Netherlands. As a result, the Dutch population is characterized by a variety of cultural backgrounds. That The Netherlands are becoming more colorful is a fact that you'd better be prepared for as a employer. But how does it feel to be working in a culturally diverse workforce? In this column we present a series of interviews with both native Dutch and non-native Dutch employees. Today, in the first part we have an interview with Ömer.

Ömer is of Turkish descent and has lived in The Netherlands for a long period of time. Before his arrival in The Netherlands he had finished his schooling and he worked for various organizations. After his arrival in The Netherlands Ömer had, just like many other non-natives, problems finding a suitable job. Recently, he got a job at Philips.

About his job at Philips, Ömer says: 'I really feel part of Philips and I am proud to be working for this company. In our department we treat each other well and we form a strong team. Within the team we feel responsible for each other and for the work that has to be done. I really feel part of this team. Whenever there is a problem, we solve it together. I also see my colleagues outside the office, we go out for dinner or do other nice things'. In response to the question what his main drive is at work, Ömer says: 'to me it is not just important that I have a job, but also in what organization. The work atmosphere is also very important to $m e$ '.

Despite the fact that Ömer has lived in The Netherlands for quite some time already, he maintains many of his Turkish habits. He says: 'Apart form my Dutch colleagues I also have many Turkish friends. Thanks to my friends and family I am well informed about the events in Turkey. I also follow the news from Turkey on the radio and television. I regularly call my family in Turkey and I try to go there at least once a year'. At first Ömer had to get used to speaking a different language. 'When I arrived here, I tried to learn the language of this country as fast as possible. Nowadays, I mostly speak Dutch in daily life. I think it is important to master this language. But still, I am happy that I also speak Turkish at home, for I would regret it if this language was lost within our family'. To conclude
Ömer says: 'It's important to me to maintain my cultural background'.

(434 words in original version)

\section{The team identity}

\section{The multicultural workforce}

Amsterdam - The Dutch labor market is becoming more and more diverse. Over past decades many foreigners have come to The Netherlands. As a result, the Dutch population is characterized by a variety of cultural backgrounds. That The Netherlands are becoming more colorful is a fact that you'd better be prepared for as a employer. But how does it feel to be working in a culturally diverse workforce? In this column we present a series of interviews with both native Dutch and non-native Dutch employees. Today, in the first part we have an interview with Ömer.

Ömer is of Turkish descent and has lived in The Netherlands for a long period of time. Before his arrival in The Netherlands he had finished his schooling and he worked for various organizations. After his arrival in The Netherlands Ömer had, just like many other non-natives, problems finding a suitable job. Recently, he got a job at Philips.

About his job at Philips, Ömer says: 'I really feel part of Philips and I am proud to be working for this company. In our department we treat each other well and we form a strong team. Within the team we feel responsible for each other and for the work that has to be done. I really feel part of this team. Whenever there is a problem, we solve it together. I also see my colleagues outside the office, we go out for dinner or do other nice things'. In response to the question what his main drive is at work, Ömer says: 'to me it is not just important that I have a job, but also in what organization. The work atmosphere is also very important to me'.

Ömer has lived in The Netherlands for quite some time and feels few ties with Turkey. He says: 'I hardly know what is going on in Turkey, on the radio and television I mostly follow the news from The Netherlands. I haven't been in Turkey for quite some time. He says: 'I mostly have Dutch friends'. At first Ömer had to get used to speaking a different language. 'When I arrived here, I tried to learn the language of this country as fast as possible. Nowadays, I mostly speak Dutch in daily life. I think it is important to master this language. The Turkish language has become less important. To conclude Ömer says: 'It's not that important to me to maintain my cultural background'.

(412 words in original version) 


\section{The cultural identity}

\section{The multicultural workforce}

Amsterdam - The Dutch labor market is becoming more and more diverse. Over past decades many foreigners have come to The Netherlands. As a result, the Dutch population is characterized by a variety of cultural backgrounds. That The Netherlands are becoming more colorful is a fact that you'd better be prepared for as a employer. But how does it feel to be working in a culturally diverse workforce? In this column we present a series of interviews with both native Dutch and non-native Dutch employees. Today, in the first part we have an interview with Ömer.

Ömer is of Turkish descent and has lived in The Netherlands for a long period of time. Before his arrival in The Netherlands he had finished his schooling and he worked for various organizations. After his arrival in The Netherlands Ömer had, just like many other non-natives, problems finding a suitable job. Recently, he got a job at Philips.

About his job at Philips, Ömer says: 'I work here for the money, really. I am satisfied with my salary at Philips, but I don't really feel committed to the company. The people in my department don't mean much to me. We can work together, but we are not a strong team. Everybody just does what he has to do, and problems are solved independently. I don't meet with colleagues outside office hours'. In response to the question what his main drive is at work, Ömer says: 'to me it is important that I have a job, but I don't care too much if it's in this organization or another one'.

Despite the fact that Omer has lived in The Netherlands for quite some time already, he maintains many of his Turkish habits. He says: 'I mostly have Turkish friends'. Thanks to my friends and family I am well informed about the events in Turkey. I also follow the news from Turkey on the radio and television. I regularly call my family in Turkey and I try to go there at least once a year'. At first Ömer had to get used to speaking a different language. 'When I arrived here, I was forced to learn Dutch as fast as possible. But, I think it's important to speak Turkish at home as much as possible, for I would regret it if this language was lost within our family'. To conclude Ömer says: 'It's important to me to maintain my cultural background'.

(393 words in original version)

\section{The marginal identity}

\section{The multicultural workforce}

Amsterdam - The Dutch labor market is becoming more and more diverse. Over past decades many foreigners have come to The Netherlands. As a result, the Dutch population is characterized by a variety of cultural backgrounds. That The Netherlands are becoming more colorful is a fact that you'd better be prepared for as a employer. But how does it feel to be working in a culturally diverse workforce? In this column we present a series of interviews with both native Dutch and non-native Dutch employees. Today, in the first part we have an interview with Ömer.

Ömer is of Turkish descent and has lived in The Netherlands for a long period of time. Before his arrival in The Netherlands he had finished his schooling and he worked for various organizations. After his arrival in The Netherlands Ömer had, just like many other non-natives, problems finding a suitable job. Recently, he got a job at Philips.

About his job at Philips, Ömer says: 'I work here for the money, really. I am satisfied with my salary at Philips, but I don't really feel committed to the company. The people in my department don't mean much to me. We can work together, but we are not a strong team. Everybody just does what he has to do, and problems are solved independently. I don't meet with colleagues outside office hours'. In response to the question what his main drive is at work, Ömer says: 'to me it is important that I have a job, but I don't care too much if it's in this organization or another one'.

Ömer has lived in The Netherlands for quite some time and feels few ties with Turkey. He says: 'I hardly know what is going on in Turkey, on the radio and television I mostly follow the world news. I haven't been in Turkey for quite some time. Ömer's friends are from everywhere, not from The Netherlands or Turkey in particular. At first Ömer had to get used to speaking a different language. 'When I arrived here, I was forced to learn Dutch as fast as possible. The Turkish language has become less important'. To conclude Ömer says: 'It's not that important to me to maintain my cultural background'.

(365 words in original version) 